\title{
Cast-in-place concrete walls: thermal comfort evaluation of one-storey housing in São Paulo State
}

\section{Vedações verticais em concreto moldadas in loco: avaliação do conforto térmico de habitações térreas no Estado de São Paulo}
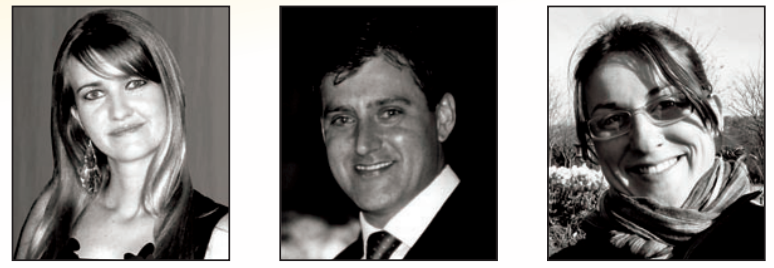

H. M. SACHT hmsacht@civil.uminho.pt

J. A. ROSSIGNOLO b rossignolo@usp.br

C. BUENO ${ }^{\circ}$ cbueno@sc.usp.br

\begin{abstract}
This paper presents a proposal of thermal performance evaluation of a one-storey housing typology (TI24A) executed by CDHU - Companhia de Desenvolvimento Habitacional e Urbano do Estado de São Paulo, considering the use of cast-in-place monolithic panels of concrete, with different thicknesses panels $\left(8,10\right.$ and $12 \mathrm{~cm}$ ) and density between 1600 and $2400 \mathrm{~kg} / \mathrm{m}^{3}$. In this study, the specific purpose was discussing the influence of the characteristic of concrete walls on the housing thermal performance without slab. Was defined of first parameters of study (definition of the one-storey housing typology, survey about housing users behavior and cities choose) and executed computational simulation (winter and summer), for four São Paulo State cities (São Paulo, São Carlos, Santos e Presidente Prudente), with the software Arquitrop 3.0 in a one-storey housing. Was observed that in winter and summer the typologies analyzed, the panels thickness variation had more influence about results than different concrete densities. The minimum level of thermal performance (M) in winter has been granted for some cities, with exception of Santos. In summer one of São Paulo city's typology was attended the minimum level of thermal performance in agreement with standard "NBR 15575 Residential buildings up to five storied - Performance, Part 1: General requirements".
\end{abstract}

Keywords: Thermal performance; Low-cost one-storey housing; Cast-in-place monolithic panels of concrete.

\section{Resumo}

Neste artigo apresenta-se a avaliação do desempenho térmico de uma tipologia habitacional térrea (TI24A) executada pela CDHU - Companhia de Desenvolvimento Habitacional e Urbano do Estado de São Paulo, utilizando o sistema construtivo de painéis monolíticos de concreto moldados in loco, com painéis de diferentes espessuras $\left(8,10\right.$ e $12 \mathrm{~cm}$ ) e massas específicas variando entre 1600 e $2400 \mathrm{~kg} / \mathrm{m}^{3}$. Analisou-se especificamente a influência dos tipos de vedação no desempenho térmico de habitações sem laje. Foram determinados os critérios iniciais de estudo (definição da tipologia habitacional térrea, levantamento de dados comportamentais dos usuários e determinação das cidades) e executadas simulações computacionais (inverno e verão), para 4 cidades do Estado de São Paulo (São Paulo, São Carlos, Santos e Presidente Prudente), empregando o software Arquitrop 3.0. Observou-se que para inverno e verão, nas tipologias térreas analisadas, a variação de massa específica do concreto pouco influenciou nos resultados, já a variação das espessuras dos painéis representou maiores diferenças nas temperaturas internas. Para o inverno foi atendido o nível mínimo de desempenho térmico para grande parte das cidades, com exceção de Santos. Para o verão, somente para a cidade de São Paulo o nível mínimo de desempenho térmico foi atendido de acordo com a norma "NBR15575-1 Edifícios habitacionais de até 5 pavimentos - Desempenho, Parte 1: Requisitos Gerais".

Palavras-chave: Desempenho térmico; Habitação térrea de interesse social; Painéis de concreto moldados in loco.

Architect and Urbanist, MSc in Architecture, Urbanism and Technology by EESC/USP, Civil Engineering PhD Student at University of Minho, hmsacht@civil.uminho.pt, Building Physics and Construction Technologies Laboratory (LFTC), Campus de Azurém, 4800-058, Guimarães, Portugal.

Associate Professor, Biossystems Engineering, University of São Paulo, School of Zootechny and Food Engineering FZEA-USP, rossignolo@usp.br, Av. Duque de Caxias Norte, 225 - ZEA/Campus da USP - 13635-900, Pirassununga-SP, Brazil.

${ }^{\circ}$ Architect and Urbanist, MSc in Architecture, Urbanism and Technology by EESC/USP, Architecture and Urbanism PhD Student at EESC/USP, cbueno@sc.usp.br, Department of Architecture and Urbanism, Sao Carlos Engineering School (EESC/USP), Av. Trabalhador São-carlense, 400, 13566-590, São Carlos - SP, Brazil. 
As condições de desempenho térmico para o verão, em grande parte dos casos, não atendeu aos requisitos mínimos da NBR 15575-1. Para a cidade de São Paulo somente a tipologia com painéis de $12 \mathrm{~cm}$ de espessura e massa específica 2400 $\mathrm{kg} / \mathrm{m}^{3}$ atendeu ao nível mínimo de desempenho (M) ( $T$ máxima interna $\leq \mathrm{T}$ máxima externa). Isso mostra que para essa estação, quase totalidade dos tipos de painéis empregados nas habitações térreas sem laje não estão adequados em relação ao desempenho térmico.

Diante dos resultados obtidos, salienta-se que deve ser evitada a recorrente reprodução de tipologias habitacionais sem uma preocupação maior com as especificidades regionais. A elaboração de projetos de habitação de interesse social adequados ao clima e as características locais, representa além dos benefícios para os próprios moradores, a melhoria dos assentamentos humanos e da qualidade de vida nas cidades brasileiras.

\section{Agradecimentos}

Os autores agradecem à instituição de fomento à pesquisa FAPESP - Fundação do Amparo à Pesquisa do Estado de São Paulo e ao Laboratório de Construção Civil (LCC) da Escola de Engenharia de São Carlos da Universidade de São Paulo (EESC - USP).

\section{Referências bibliográficas}

[01] LORDSLEEM JUNIOR, A. C. et al. Estágio atual do uso de paredes maciças moldadas no local em São Paulo. In: CONGRESSO LATINO AMERICANO TECNOLOGIA E GESTÃO NA PRODUÇÃO DE EDIFÍCIOS - SOLUÇÕES PARA O TERCEIRO MILÊNIO, 1998, São Paulo.

Anais... São Paulo: Escola Politécnica, Universidade de São Paulo - Departamento de Engenharia Civil PCC. nov, 1998.

[02] LORDSLEEM JÚNIOR, A. C. O processo de produção das paredes maciças. In: SEMINÁRIO TECNOLOGIA E GESTÃO NA PRODUÇÃO DE EDIFÍCIOS VEDAÇÕES VERTICIAS - TECNOLOGIA E GESTÃO NA PRODUÇÃO DE EDIFÍCIOS, 1998, São Paulo. Anais... São Paulo: Escola Politécnica da Universidade de São Paulo, Departamento de Engenharia de Construção Civil - PCC, jul. 1998 b.

[03] ABIKO, A.K.; ORNSTEIN, S. W. (Organizadores). Inserção Urbana e Avaliação Pós-Ocupação (APO) da Habitação de Interesse Social. Coletânea Habitare/ Finep, 1São Paulo: FAUUSP. 2002. 373p.

[04] MORELLO, A.; SATTLER, M. A. Avaliação do desempenho térmico de um protótipo habitacional construído na cidade de Porto Alegre - Período de inverno de 2003. . In: I Conferência Latino-Americana de Construção Sustentável. X Encontro Nacional de Tecnologia do Ambiente Construído. São Paulo, julho de 2004.

[05] VITTORINO, F.; AKUTSU, M. Validação de softwares aplicativos para simulação do comportamento térmico de habitações. São Paulo: IPT, 2004. (IPT, relatório técnico, 72).
[06] RAUBER, D.L., VAN BELLEN, II.M., SCHUCH, L.M.S., NARCISO FILHO, P. A. L.; LAMBERTS, R. Comparação de softwares de análise térmica de edificações : Resultados para um projeto padrão e interface programa - usuário. In: II Encontro Nacional de Conforto no Ambiente Construído ENCAC. Florianópolis: Anais... ENCAC, 1993.

[07] ASSOCIAÇÃO BRASILEIRA DE NORMAS TÉCNICAS (ABNT). NBR 15575-1: Edifícios habitacionais de até 5 pavimentos - Desempenho Parte 1: Requisitos Gerais. Rio de Janeiro, 2008.

[08] ASSOCIAÇÃO BRASILEIRA DE NORMAS TÉCNICAS (ABNT). NBR 15220: Desempenho térmico de edificações. Parte 3: Zoneamento Bioclimático Brasileiro e Diretrizes para Habitações Unifamiliares de Interesse Social. Rio de Janeiro, 2005.

[09] COMPANHIA DE DESENVOLVIMENTO HABITACIONAL E URBANO DO ESTADO DE SÃO PAULO (CDHU). Padrão Habitacional TI24A - Arquivo de Auto CAD. São Paulo, 2007.

[10] (CDHU). Manual Técnico de Projetos. São Paulo, 1998.

[11] (CDHU). Memorial Descritivo/Especificações Técnicas - Unidades Habitacionais Térreas TI24A/ TG23A/ TI24C/ TI23C/ TI23D/ TG22B-01. São Paulo, 2005.

[12] RORIZ, M. (Programação); BASSO, A. (Dados). ARQUITROP Versão 3.0: Conforto térmico e economia de energia nas edificações. Sistema integrado de rotinas e bancos de dados para apoio às atividades de projeto em Arquitetura e Engenharia Civil. São Paulo: UFSCAR, 1989.

[13] PÉREZ, J. P. H.; ROMULUS, A. M. Thermodynamique. Fondements et Applications. Masson, Paris, 1993.

[14] SACHT, H. M. Painéis de vedação de concreto moldados in loco: Avaliação de desempenho térmico e desenvolvimento de concretos. Dissertação (Mestrado). Universidade de São Paulo, Escola de Engenharia de São Carlos USP/ EESC, 2008.

[15] SACHT, H. M.; ROSSIGNOLO, J. A. Habitações térreas de interesse social: o uso e vedações em concreto com diferentes massas específicas e as condições de conforto térmico no estado de São Paulo. In: Anais... NUTAU 2008 Seminário Internacional, O Espaço Sustentável - Inovações em Edifícios e Cidades. São Paulo, 2008.

[16] GIVONI, B. (1992). Comfort Climate Analysis and Building Design Guidelines. Energy and Buildings, v. 18, n. 1, p. 11-23.

[17] ROSSIGNOLO, J. A. Levantamento Fotográfico em Santa Maria da Serra - SP, 2006. 